\title{
Egg Parasitic Fungus, Engyodontium aranearum with other Biocontrol Agents, Organic Amendment and Carbofuran for the Management of Meloidogyne incognita on Tomato
}

\author{
M. Muthulakshmi*, B. Anita and S. Subramanian \\ Department of Nematology, Tamil Nadu Agricultural University, \\ Coimbatore - 641 003, India \\ *Corresponding author
}

\begin{tabular}{|c|c|}
\hline & A B S T R A C T \\
\hline & \multirow{4}{*}{$\begin{array}{l}\text { The effect of Engyodontium aranearum, a nematode egg parasitic fungus, alone } \\
\text { and along with other biocontrol agents, organic amendment and carbofuran was } \\
\text { evaluated in pot culture infested with Meloidogyne incognita on tomato under pot } \\
\text { and field condition for two seasons. Plant growth parameters, fruit yield, root } \\
\text { population and final nematode population were determined at harvest. The results } \\
\text { revealed that all the treatments were effective in increasing the plant growth with } \\
\text { significant reductions in nematode populations. Among the treatments, the } \\
\text { combined soil application of E. aranearum with } P \text {. flouroscens each at } 2.5 \mathrm{~kg} / \mathrm{ha} \\
\text { increased the tomato yield by } 55.68 \text { per cent under pot culture conditions and } \\
41.62 \text { per cent under field conditions. The soil nematode population was decreased } \\
\text { by } 54.74 \text { per cent under pot culture and } 60.75 \text { under field conditions. There was } \\
\text { also significant reduction in nematode population in roots. }\end{array}$} \\
\hline $\begin{array}{l}\text { Engyodontium } \\
\text { aranearum, } \\
\text { Biocontrol agents, } \\
\text { P. flouroscens, Root } \\
\text { knot nematode, } \\
\text { Meloidogyne } \\
\text { incognita, Tomato. }\end{array}$ & \\
\hline Article Info & \\
\hline $\begin{array}{l}\text { Accepted: } \\
\text { 04 April } 2017 \\
\text { Available Online: } \\
10 \text { May } 2017\end{array}$ & \\
\hline
\end{tabular}

\section{Introduction}

India's diverse climate ensures availability of all varieties of fresh fruits and vegetables. It ranks second in vegetables production in the world, after China. India produced 146.554 million metric tonnes of vegetable from 8.495 million hectares. Tomato is the world's largest and important commercial vegetable grown in tropical and subtropical areas for its fleshy fruits, taste and nutritive value. It is a short duration crop and gives high yield. In India, tomato occupies second position amongst the vegetable crops in terms of production. Total production of tomato in the country was $17.4 \mathrm{mt}$ from an area of $0.87 \mathrm{mha}$ (http://www.dacnet.nic.in). In Tamil
$\mathrm{Nadu}$, tomato occupies an area of 0.02 mha with production of $0.29 \mathrm{mt}$ (http://www. tn.gov.in).

Tomato is affected adversely by several pests, diseases and nematodes, which result in heavy yield losses. Plant parasitic nematodes are the major pests of agriculture throughout the world, which cause 12.3 per cent yield losses (Sassar and Freckman, 1987) with a global economic impact of more than $\$ 125$ billion annually worldwide (Chitwood, 2003). Among crop plants, vegetables are highly susceptible to root knot nematodes. The southern root knot nematode, Meloidogyne 
incognita is one of the major constraints in the production of tomato in tropical and subtropical regions. In India, the annual losses caused by root knot nematode, $M$. incognita are 27.2 per cent in tomato (Jain et al., 2007). Present strategies for nematode management largely depend on cultural practices, use of resistant varieties and chemical applications often in combinations (Hague and Gowen, 1987). A large number of beneficial organisms including fungi, bacteria, viruses and predatory nematodes have been found to parasitize the infective juveniles, female and eggs of root knot and cyst nematodes (Stirling, 1991).

The egg parasitic fungi, Pochonia chlamydosporia and Paecilomyces lilaciuns have been associated with soils which suppress the multiplication of cyst nematode populations (Kerry et al., 1993). The egg parasitic fungus, Engyodontium aranearum parasitization of the potato cyst nematodes was first reported from The Nilgiris (Muthulakshmi, 2011). Hence, the present investigation was under taken with Egg parasitic fungus, Engyodontium aranearum alone and with other biocontrol agents, organic amendment and carbofuran in tomato under pot and field conditions to assess the effectiveness against Meloidogyne incognita.

\section{Materials and Methods}

The compatibility of E. aranearum with other biocontrol agents viz., Trichoderma viride, $P$. lilacinus, Pseudomonas fluorescens and organic amendment, neem cake and carbofuran was studied under pot culture and field conditions.

Soil application of $E$. aranearum against $M$. incognita was carried out under pot culture conditions in tomato. Tomato seeds (cv. Co 3) were sown in pots and maintained for 25 days. After that seedlings were transplanted in pots containing pot mixture. The tomato plants were inoculated @ one $\mathrm{J}_{2} / \mathrm{g}$ of soil on 15 days after transplanting. The experiment was conducted under glasshouse conditions in a completely randomized design (CRD) with the following treatments which were replicated three times.

$1-$ E. aranearum @ $2.5 \mathrm{~kg} / \mathrm{ha}$

2-E. aranearum@2.5 kg/ha + P. lilacinus @ $2.5 \mathrm{~kg} / \mathrm{ha}$

$3-$ E. aranearum @ $2.5 \mathrm{~kg} / \mathrm{ha}+T$.viride@ $2.5 \mathrm{~kg} / \mathrm{ha}$

4 - E. aranearum @ $2.5 \mathrm{~kg} / \mathrm{ha}+P$. fluorscens @ $2.5 \mathrm{~kg} / \mathrm{ha}$

5- E. aranearum @2.5 kg/ha + Neem cake $(250 \mathrm{~kg} / \mathrm{ha})$

6 - E. aranearum @ $2.5 \mathrm{~kg} / \mathrm{ha}+$ Carbofuran $3 \mathrm{G} @ 1 \mathrm{~kg}$ a.i./ha

7 - Control

Two field experiments were conducted in nematode sick field of Madhampatti and Karadimadai village, Coimbatore district, Tamil Nadu to evaluate $E$. aranearum formulation against $M$. incognita in tomato (cv. Co3) and in bhendi (Mahyco hybrid No. 10) respectively. The experiment was conducted in a randomized block design (RBD) with treatments and replications similar to glasshouse experiment.

Plant growth parameters viz., shoot length, shoot weight, root length, root weight and yield were observed at after harvest. The observations on soil and root population of root knot nematode, number of females $/ 5 \mathrm{~g}$ root, number of egg masses $/ 5 \mathrm{~g}$ root and root knot index were recorded at after harvest.

The data from the experiments were subjected to statistical analysis. The treatment means were compared by Duncan's Multiple Range Test (DMRT) (Gomez and Gomez, 1984). The package used for analysis was IRRISTAT version 92-1 by International Rice 
Research Institute, Biometrics Unit, seed treatment with combination of $P$. Philippines.

\section{Results and Discussion}

\section{Pot culture experiment}

In tomato, E. aranearum significantly improved plant growth parameters when applied either alone or in combination with other biocontrol agents, organic amendments and carbofuran. The highest shoot length $(66.39 \mathrm{~cm})$, root length $(17.62 \mathrm{~cm})$, shoot weight $(22.67 \mathrm{~g})$, root weight $(13.48 \mathrm{~g})$ and yield (628.37 g/plant) were recorded in plants treated with $E$. aranearum $(2.5 \mathrm{~kg} / \mathrm{ha})+P$. fluorescens $(2.5 \mathrm{~kg} / \mathrm{ha})$ compared to $40.47 \mathrm{~cm}$, $10.54 \mathrm{~cm}, 12.98 \mathrm{~g}, 7.85 \mathrm{~g}$ and $403.62 \mathrm{~g} / \mathrm{plant}$, shoot length, root length, shoot weight, root weight and yield respectively in untreated control (Table 1). There was a significant decrease in no. of females, number of egg masses, root knot index, root population and soil population in both the combined and individual treatments compared to untreated control.

The combined application of $E$. aranearum $(2.5 \mathrm{~kg} / \mathrm{ha})+P$. fluorescens $(2.5 \mathrm{~kg} / \mathrm{ha})$ reduced the number of females and number of egg masses by 51.53 and 61.33 per cent over control. There was also 52.00 and 54.74 percent reduction in the nematode population in roots and soil (Table 2). These findings were in agreement with the egg parasitic fungus, $P$. lilacinus in combination with $V$. chlamydosporium significantly increased the length and weight of root and shoot and reduced root galling in okra plant (Sobita Simon and Avinash Pandey, 2010).

Kumar and Prabhu (2008) reported that combined application of $T$. harzianum $+P$. chlamydosporia significantly reduced the cyst nematode $H$. cajani population in pigeon pea. Latha and Narashimhan (2001) reported that

fluorescens, $T$. viride and $P$. lilacinus reduced the infestation of $M$. phaseolina and $H$. cajani complex in black gram.

\section{Field experiments}

In tomato, the highest shoot length (73.48 $\mathrm{cm})$, root length $(35.52 \mathrm{~cm})$, shoot weight $(28.52 \mathrm{~g})$, root weight $(17.38 \mathrm{~g})$ and yield (24.67 t/ha) was recorded in plants treated with E. aranearum $(2.5 \mathrm{~kg} / \mathrm{ha})+P$. fluorescens $(2.5 \mathrm{~kg} / \mathrm{ha})$ compared to 45.57 $\mathrm{cm}, 21.84 \mathrm{~cm}, 17.58 \mathrm{~g}, 11.54 \mathrm{~g}$ and 17.42 t/ha, shoot length, root length, shoot weight, root weight and yield respectively in untreated control (Table 3).

There was a significant decrease in no. of females, no. of egg masses, root knot index, root population and soil population in both the combined and individual treatments compared to untreated control.

The combined application of E. aranearum $(2.5 \mathrm{~kg} / \mathrm{ha})+P$. fluorescens $(2.5 \mathrm{~kg} / \mathrm{ha})$ reduced the number of females and number of egg masses by 63.47 and 61.85 per cent over control. There was also 61.95 and 60.75 per cent reduction in the nematode population in roots and soil. These findings were in agreement with the Gopinatha et al., (2002) obtained significant increase in plant growth and yield of tomato and reduction in root galling in combinations of $V$. chlamydosporium + carbofuran, marigold + carbofuran, V. chlamydosporium + marigold (Table 4).

The combined application of $P$. chlamydosporia with neem cake and/or carbofuran at reduced doses gave better recovery in comparison to either of the single application in terms of shoot length, shoot weight, root length and fruit yield. 
Table.1 Compatibility of E. aranearum with other biocontrol agents, organic amendments and carbofuran on plant tomato under pot culture conditions

\begin{tabular}{|c|c|c|c|c|c|}
\hline Treatments & $\begin{array}{l}\text { Shoot length } \\
(\mathrm{cm})\end{array}$ & $\begin{array}{l}\text { Root length } \\
(\mathrm{cm})\end{array}$ & $\begin{array}{c}\text { Shoot } \\
\text { weight }(g)\end{array}$ & $\begin{array}{l}\text { Root weight } \\
\text { (g) }\end{array}$ & $\begin{array}{c}\text { Yield } \\
\text { (g/plant) }\end{array}$ \\
\hline E. aranearum@2.5 kg/ha & $\begin{array}{c}53.54^{\mathrm{e}} \\
(32.30)\end{array}$ & $\begin{array}{l}14.19^{\mathrm{d}} \\
(34.63)\end{array}$ & $\begin{array}{l}18.46^{\mathrm{d}} \\
(42.22)\end{array}$ & $\begin{array}{c}10.64^{\mathrm{e}} \\
(35.54)\end{array}$ & $\begin{array}{l}536.59^{\mathrm{d}} \\
(32.94)\end{array}$ \\
\hline $\begin{array}{l}\text { E. aranearum@2.5 kg/ha }+P \text {. lilacinus } \\
@ 2.5 \mathrm{~kg} / \mathrm{ha}\end{array}$ & $\begin{array}{l}58.78^{\mathrm{cd}} \\
(45.24) \\
\end{array}$ & $\begin{array}{l}15.38^{c} \\
(45.92) \\
\end{array}$ & $\begin{array}{l}19.30^{c} \\
(48.70) \\
\end{array}$ & $\begin{array}{l}11.42^{\mathrm{d}} \\
(45.48) \\
\end{array}$ & $\begin{array}{l}550.38^{d} \\
(36.36)\end{array}$ \\
\hline $\begin{array}{l}\text { E.aranearum@2.5 kg/ha +T.viride @ } \\
2.5 \mathrm{~kg} / \mathrm{ha}\end{array}$ & $\begin{array}{l}58.42^{d} \\
(44.35)\end{array}$ & $\begin{array}{l}15.30^{c} \\
(45.16)\end{array}$ & $\begin{array}{l}19.23^{\mathrm{c}} \\
(48.15)\end{array}$ & $\begin{array}{l}11.63^{d} \\
(48.15)\end{array}$ & $\begin{array}{l}554.62^{d} \\
(37.41)\end{array}$ \\
\hline $\begin{array}{l}\text { E. aranearum@2.5 kg/ha }+P \text {. } \\
\text { fluorscens@ } 2.5 \mathrm{~kg} / \mathrm{ha}\end{array}$ & $\begin{array}{l}66.39^{\mathrm{a}} \\
(64.05)\end{array}$ & $\begin{array}{l}17.62^{\mathrm{a}} \\
(67.17)\end{array}$ & $\begin{array}{l}22.67^{\mathrm{a}} \\
(74.65)\end{array}$ & $\begin{array}{l}13.48^{\mathrm{a}} \\
(71.72)\end{array}$ & $\begin{array}{l}628.37^{\mathrm{a}} \\
(55.68)\end{array}$ \\
\hline $\begin{array}{l}\text { E.aranearum@ } 2.5 \mathrm{~kg} / \mathrm{ha}+\text { Neem cake } \\
(250 \mathrm{~kg} / \mathrm{ha})\end{array}$ & $\begin{array}{l}60.73^{\mathrm{c}} \\
(50.06)\end{array}$ & $\begin{array}{l}15.54^{\mathrm{c}} \\
(47.44)\end{array}$ & $\begin{array}{l}19.86^{\mathrm{c}} \\
(53.00)\end{array}$ & $\begin{array}{l}12.46^{\mathrm{c}} \\
(58.73)\end{array}$ & $\begin{array}{l}585.61^{\mathrm{c}} \\
(45.09)\end{array}$ \\
\hline $\begin{array}{l}\text { E. aranearum@ } 2.5 \mathrm{~kg} / \mathrm{ha}+\text { Carbofuran } \\
3 \mathrm{G} @ 1 \mathrm{~kg} \text { a.i./ha }\end{array}$ & $\begin{array}{l}64.72^{b} \\
(59.92) \\
\end{array}$ & $\begin{array}{l}17.04^{\mathrm{b}} \\
(61.67) \\
\end{array}$ & $\begin{array}{l}21.38^{b} \\
(64.71) \\
\end{array}$ & $\begin{array}{l}13.04^{\mathrm{b}} \\
(66.11) \\
\end{array}$ & $\begin{array}{l}607.38^{b} \\
(50.48) \\
\end{array}$ \\
\hline Control & $40.47^{\mathrm{f}}$ & $10.54^{\mathrm{e}}$ & $12.98^{\mathrm{e}}$ & $7.85^{\mathrm{f}}$ & $403.62^{\mathrm{e}}$ \\
\hline SEd & 0.9479 & 0.2486 & 0.3160 & 0.1899 & 9.0880 \\
\hline $\mathrm{CD}(\mathrm{P}=0.05)$ & 2.0332 & 0.5332 & 0.6778 & 0.4073 & 19.4940 \\
\hline
\end{tabular}

Values are mean of three replications. Column figures followed by different letters are significant from each other at 5 per cent level by DMRT 
Table.2 Effect of E. aranearum alone and along with other biocontrol agents, organic amendments and carbofuran on tomato infested with $M$. incognita under pot culture conditions

\begin{tabular}{|c|c|c|c|c|c|}
\hline Treatments & $\begin{array}{c}\text { No. of } \\
\text { females } \\
(5 \mathrm{~g} \text { root })\end{array}$ & $\begin{array}{l}\text { No. of egg } \\
\text { mass } \\
\text { root })\end{array}$ & $\begin{array}{l}\text { Root knot } \\
\text { index }\end{array}$ & $\begin{array}{c}\text { Root } \\
\text { population } \\
\text { (5g root) }\end{array}$ & $\begin{array}{l}\text { Soil } \\
\text { population } \\
\text { (250cc soil) }\end{array}$ \\
\hline E. aranearum@2.5 kg/ha & $\begin{array}{l}63.82^{\mathrm{e}} \\
(33.81)\end{array}$ & $\begin{array}{l}29.27^{\mathrm{e}} \\
(41.89)\end{array}$ & 2.33 & $\begin{array}{l}81.73^{\mathrm{e}} \\
(34.99)\end{array}$ & $\begin{array}{l}111.54^{d} \\
(45.49)\end{array}$ \\
\hline $\begin{array}{l}\text { E. aranearum@2.5 kg/ha }+ \\
P . \text { lilacinus @ } 2.5 \mathrm{~kg} / \mathrm{ha}\end{array}$ & $\begin{array}{l}60.41^{d} \\
(37.34)\end{array}$ & $\begin{array}{l}26.98^{\mathrm{d}} \\
(46.44)\end{array}$ & 2.00 & $\begin{array}{l}77.93^{d} \\
(38.01)\end{array}$ & $\begin{array}{l}106.58^{\mathrm{c}} \\
(47.92)\end{array}$ \\
\hline $\begin{array}{l}\text { E. aranearum@2.5 kg/ha + } \\
\text { T. viride@ } 2.5 \mathrm{~kg} / \mathrm{ha}\end{array}$ & $\begin{array}{l}60.53^{d} \\
(37.22)\end{array}$ & $\begin{array}{l}26.76^{\mathrm{d}} \\
(46.87)\end{array}$ & 2.00 & $\begin{array}{l}76.47^{\mathrm{d}} \\
(39.17)\end{array}$ & $\begin{array}{l}106.83^{c} \\
(47.80)\end{array}$ \\
\hline $\begin{array}{l}\text { E. aranearum@2.5 kg/ha + } \\
P . \text { fluorscens @ } 2.5 \mathrm{~kg} / \mathrm{ha}\end{array}$ & $\begin{array}{l}46.73^{\mathrm{a}} \\
(51.53)\end{array}$ & $\begin{array}{l}19.48^{\mathrm{a}} \\
(61.33)\end{array}$ & 1.33 & $\begin{array}{l}60.35^{\mathrm{a}} \\
(52.00)\end{array}$ & $\begin{array}{l}92.63^{\mathrm{a}} \\
(54.74)\end{array}$ \\
\hline $\begin{array}{l}\text { E. aranearum@2.5 kg/ha + } \\
\text { Neem cake }(250 \mathrm{~kg} / \mathrm{ha})\end{array}$ & $\begin{array}{l}57.48^{c} \\
(40.39)\end{array}$ & $\begin{array}{l}23.52^{c} \\
(53.31)\end{array}$ & 2.00 & $\begin{array}{l}69.32^{c} \\
(44.86)\end{array}$ & $\begin{array}{l}102.62^{c} \\
(49.85)\end{array}$ \\
\hline $\begin{array}{l}\text { E. aranearum@ } 2.5 \mathrm{~kg} / \mathrm{ha}+ \\
\text { Carbofuran 3G @ } 1 \mathrm{~kg} \\
\text { a.i./ha }\end{array}$ & $\begin{array}{l}49.39^{b} \\
(48.78)\end{array}$ & $\begin{array}{l}21.83^{b} \\
(56.66)\end{array}$ & 1.67 & $\begin{array}{l}64.48^{\mathrm{b}} \\
(48.71)\end{array}$ & $\begin{array}{l}97.38^{\mathrm{b}} \\
(52.41)\end{array}$ \\
\hline Control & $96.42^{f}$ & $50.37^{\mathrm{f}}$ & 4.33 & $125.72^{\mathrm{f}}$ & $204.64^{\mathrm{e}}$ \\
\hline SEd & 1.0447 & 0.4897 & - & 1.3378 & 2.0058 \\
\hline $\mathrm{CD}(\mathrm{P}=0.05)$ & 2.2408 & 1.0504 & - & 2.8696 & 4.3025 \\
\hline
\end{tabular}

Values are mean of three replications. Column figures followed by different letters are significant from each other at 5 per cent level by DMRT 
Table.3 Effect of E. aranearum alone and along with other biocontrol agents, organic amendments and carbofuran on plant growth parameters in tomato under field contidions

(Pooled data from two field experiments)

\begin{tabular}{|c|c|c|c|c|c|}
\hline Treatments & $\begin{array}{c}\text { Shoot } \\
\text { length } \\
\text { (cm) }\end{array}$ & $\begin{array}{c}\text { Root } \\
\text { length } \\
(\mathrm{cm})\end{array}$ & $\begin{array}{c}\text { Shoot } \\
\text { weight }(g)\end{array}$ & $\begin{array}{c}\text { Root } \\
\text { weight }(g)\end{array}$ & Yield (t/ha) \\
\hline E.aranearum@2.5 kg/ha & $\begin{array}{l}62.42^{\mathrm{e}} \\
(36.98)\end{array}$ & $\begin{array}{l}30.62^{f} \\
(40.20)\end{array}$ & $\begin{array}{l}24.38^{f} \\
(38.68)\end{array}$ & $\begin{array}{l}15.26^{\mathrm{f}} \\
(32.24)\end{array}$ & $\begin{array}{l}21.73^{f} \\
(24.74)\end{array}$ \\
\hline $\begin{array}{l}\text { E. aranearum@2.5 kg/ha }+P . \\
\text { lilacinus @ } 2.5 \mathrm{~kg} / \mathrm{ha}\end{array}$ & $\begin{array}{l}65.43^{\mathrm{d}} \\
(43.58)\end{array}$ & $\begin{array}{l}32.16^{\mathrm{e}} \\
(47.25)\end{array}$ & $\begin{array}{l}25.89^{d} \\
(47.27)\end{array}$ & $\begin{array}{l}15.86^{\mathrm{e}} \\
(37.44)\end{array}$ & $\begin{array}{l}22.65^{\mathrm{e}} \\
(30.02)\end{array}$ \\
\hline $\begin{array}{l}\text { E. aranearum@ } 2.5 \mathrm{~kg} / \mathrm{ha}+T \text {. } \\
\text { viride@ } 2.5 \mathrm{~kg} / \mathrm{ha}\end{array}$ & $\begin{array}{l}65.72^{d} \\
(44.22)\end{array}$ & $\begin{array}{r}32.42^{\mathrm{d}} \\
(48.44)\end{array}$ & $\begin{array}{l}25.75^{\mathrm{e}} \\
(46.47)\end{array}$ & $\begin{array}{l}15.93^{\mathrm{d}} \\
(38.04)\end{array}$ & $\begin{array}{l}22.78^{d} \\
(30.77)\end{array}$ \\
\hline $\begin{array}{l}\text { E. aranearum@ } 2.5 \mathrm{~kg} / \mathrm{ha}+P . \\
\text { fluorscens @ } 2.5 \mathrm{~kg} / \mathrm{ha}\end{array}$ & $\begin{array}{l}73.48^{\mathrm{a}} \\
(61.25)\end{array}$ & $\begin{array}{l}35.52^{\mathrm{a}} \\
(62.64)\end{array}$ & $\begin{array}{l}28.52^{\mathrm{a}} \\
(62.23)\end{array}$ & $\begin{array}{l}17.38^{\mathrm{a}} \\
(50.61)\end{array}$ & $\begin{array}{l}24.67^{\mathrm{a}} \\
(41.62)\end{array}$ \\
\hline $\begin{array}{l}\text { E. aranearum@ } 2.5 \mathrm{~kg} / \mathrm{ha}+\mathrm{Neem} \\
\text { cake }(250 \mathrm{~kg} / \mathrm{ha})\end{array}$ & $\begin{array}{l}69.35^{\mathrm{c}} \\
(52.18)\end{array}$ & $\begin{array}{l}33.48^{\mathrm{c}} \\
(53.30)\end{array}$ & $\begin{array}{l}26.85^{\mathrm{c}} \\
(52.73)\end{array}$ & $\begin{array}{l}16.12^{c} \\
(39.69)\end{array}$ & $\begin{array}{l}23.56^{\mathrm{c}} \\
(35.25)\end{array}$ \\
\hline $\begin{array}{l}\text { E. aranearum@ } 2.5 \mathrm{~kg} / \mathrm{ha}+ \\
\text { Carbofuran 3G @ } 1 \mathrm{~kg} \text { a.i./ha }\end{array}$ & $\begin{array}{l}71.43^{b} \\
(56.75)\end{array}$ & $\begin{array}{l}34.64^{\mathrm{b}} \\
(58.61)\end{array}$ & $\begin{array}{l}27.74^{\mathrm{b}} \\
(57.79)\end{array}$ & $\begin{array}{l}16.46^{\mathrm{b}} \\
(42.63)\end{array}$ & $\begin{array}{l}24.14^{\mathrm{b}} \\
(38.58)\end{array}$ \\
\hline Control & $45.57^{\mathrm{f}}$ & $21.84^{\mathrm{g}}$ & $17.58^{\mathrm{g}}$ & $11.54^{\mathrm{g}}$ & $17.42^{\mathrm{g}}$ \\
\hline SEd & 0.1522 & 0.0735 & 0.0597 & 0.0311 & 0.0385 \\
\hline $\mathrm{CD}(\mathrm{P}=0.05)$ & 0.3317 & 0.1601 & 0.1300 & 0.0677 & 0.0839 \\
\hline
\end{tabular}

Values are mean of three replications. Column figures followed by different letters are significant from each other at 5 per cent level by DMRT 
Table.4 Effect of E. aranearum alone and along with other biocontrol agents, organic amendments and carbofuran on root knot nematodes in tomato under field conditions

\begin{tabular}{|c|c|c|c|c|c|}
\hline \multicolumn{6}{|c|}{ (Pooled data from two field experiments) } \\
\hline Treatments & $\begin{array}{c}\text { No. of } \\
\text { females } \\
\text { (5g root) }\end{array}$ & $\begin{array}{c}\text { No. of egg } \\
\text { mass } \\
(5 \mathrm{~g} \text { root })\end{array}$ & $\begin{array}{l}\text { Root knot } \\
\text { index }\end{array}$ & $\begin{array}{c}\text { Root } \\
\text { population } \\
\text { (5g root) }\end{array}$ & $\begin{array}{c}\text { Soil } \\
\text { population } \\
\text { (250cc soil) }\end{array}$ \\
\hline E. aranearum@2.5 kg/ha & $\begin{array}{l}72.12^{\mathrm{f}} \\
(34.66)\end{array}$ & $\begin{array}{l}35.64^{\mathrm{f}} \\
(33.57)\end{array}$ & 2.33 & $\begin{array}{l}124.54^{\mathrm{f}} \\
(34.55)\end{array}$ & $\begin{array}{l}147.38^{f} \\
(40.05)\end{array}$ \\
\hline $\begin{array}{l}\text { E. aranearum@ } 2.5 \mathrm{~kg} / \mathrm{ha}+P . \\
\text { lilacinus@ } 2.5 \mathrm{~kg} / \mathrm{ha}\end{array}$ & $\begin{array}{l}66.26^{\mathrm{e}} \\
(39.97)\end{array}$ & $\begin{array}{l}31.48^{\mathrm{e}} \\
(41.32)\end{array}$ & 2.00 & $\begin{array}{l}112.53^{\mathrm{e}} \\
(40.86)\end{array}$ & $\begin{array}{l}130.84^{\mathrm{e}} \\
(46.78)\end{array}$ \\
\hline $\begin{array}{l}\text { E. aranearum@ } 2.5 \mathrm{~kg} / \mathrm{ha}+T \text {. } \\
\text { viride@2.5 kg/ha }\end{array}$ & $\begin{array}{l}65.39^{d} \\
(40.75)\end{array}$ & $\begin{array}{l}30.68^{\mathrm{d}} \\
(42.81)\end{array}$ & 2.00 & $\begin{array}{l}110.47^{\mathrm{d}} \\
(41.94)\end{array}$ & $\begin{array}{l}128.62^{d} \\
(47.68)\end{array}$ \\
\hline $\begin{array}{l}\text { E. aranearum@2.5 kg/ha +P. } \\
\text { fluorscens @ } 2.5 \mathrm{~kg} / \mathrm{ha}\end{array}$ & $\begin{array}{l}40.32^{\mathrm{a}} \\
(63.47)\end{array}$ & $\begin{array}{l}20.47^{\mathrm{a}} \\
(61.85)\end{array}$ & 1.33 & $\begin{array}{l}72.41^{\mathrm{a}} \\
(61.95)\end{array}$ & $\begin{array}{l}96.48^{\mathrm{a}} \\
(60.75)\end{array}$ \\
\hline $\begin{array}{l}\text { E. aranearum@ } 2.5 \mathrm{~kg} / \mathrm{ha}+\text { Neem } \\
\text { cake }(250 \mathrm{~kg} / \mathrm{ha})\end{array}$ & $\begin{array}{l}54.74^{\mathrm{c}} \\
(50.40)\end{array}$ & $\begin{array}{l}27.93^{\mathrm{c}} \\
(47.94)\end{array}$ & 2.00 & $\begin{array}{l}86.72^{c} \\
(54.43)\end{array}$ & $\begin{array}{l}109.52^{c} \\
(55.45)\end{array}$ \\
\hline $\begin{array}{l}\text { E. aranearum@ } 2.5 \mathrm{~kg} / \mathrm{ha}+ \\
\text { Carbofuran 3G @ } 1 \mathrm{~kg} \text { a.i./ha }\end{array}$ & $\begin{array}{l}45.38^{\mathrm{b}} \\
(58.88)\end{array}$ & $\begin{array}{l}23.28^{b} \\
(43.39)\end{array}$ & 1.67 & $\begin{array}{c}75.36^{b} \\
(60.40)\end{array}$ & $\begin{array}{l}99.73^{b} \\
(59.43)\end{array}$ \\
\hline Control & $110.37^{\mathrm{g}}$ & $53.65^{\mathrm{g}}$ & 4.33 & $190.28^{\mathrm{g}}$ & $245.84^{\mathrm{g}}$ \\
\hline SEd & 0.3779 & 0.1761 & - & 0.6615 & 0.8407 \\
\hline $\mathrm{CD}(\mathrm{P}=0.05)$ & 0.8233 & 0.3836 & - & 1.4412 & 1.8317 \\
\hline
\end{tabular}

Values are mean of three replications. Column figures followed by different letters are significant from each other at 5 per cent level by DMRT 
The combination of $P$. chlamydosporia, carbofuran and neem cake gave highest yield of okra and suppressed root knot nematode severity in terms of galling, egg production and nematode population with a marginal difference with dual application of biocontrol agent and carbofuran in okra (Dhawan and Satyendra Singh, 2009). Cannayane and Rajendran (2001) reported that single applications of either $P$. lilacinus, $P$. chalmydosporia or oil cakes suppressed $M$. incognita. Nagesh et al., (2000) reported that combined application of $P$. lilacinus $+T$. harzianum + neem cake effectively controlled $M$. incognita in gladiolus. P. fluorescens with T. viride, B. subtilis, P. lilacinus and VAM reduced the population of $R$. similis, $P$. coffeae and Helicotylenchus multicinctus in banana cv. Robusta (Shanthi et al., 2003). Rao (2005) studied the bio-efficacy and compatibility of $P$. chlamydosporia and $P$. lilacinus on $M$. javanica infecting nursery seedlings of acid lime and found that application of 5 or $10 \mathrm{~g} / \mathrm{kg}$ soil of each bioagent significantly reduced the root galling and number of nematodes in roots.

\section{Acknowledgements}

The authors are thankful to Societal Research Fellowship Scheme (WOS-B), Department of Science and Technology, New Delhi who provided necessary funds for operating the scheme work.

\section{References}

Cannayane, I. and Rajendran, G. 2001. Application of biocontrol agents and oil cakes for the management of Meloidogyne incognita in brinjal. Curr. Nematol., 12: 51-55.

Chitwood, D.J. 2003. Research on plant parasitic nematodebiology conducted by the United State Department ofAgriculture-Agriculture Research
Service. Pest Management Sci., 59: 748-753.

Dhawan, S.C. and Satyendra Singh. 2009. Compatibility of Pochonia chlamydosporia with nematicide and neem cake against root knot nematode, Meloidogyne incognita infesting okra. Indian J. Nematol., 39: 85-89.

Gomez, K.A. and Gomez, A.A. 1984. Statistical procedures for agricultural research, second edn. Wiley, New York, US.

Gopinatha, K.V., Nanjegowda, D. and Nagesh, M. 2002. Management of root knot nematode, Meloidogyne incognita on tomato using bio-agent Verticillium chlamydosporium, neem cake, marigold and carbofuran. Indian J. Nematol., 32: 179-181.

Hague, N.G.M. and Gowen, S.R. 1987. Chemical control of nematodes. In: Principles and Practice of Nematode Control in Crops. Brown, R.H., Kerry, B.R. (Eds.). Australia Academic Press, pp. 133-178.

Jain, R.K., Mathur, K.N. and Singh, R.V. 2007. Estimation oflosses due to Plant Parasitic Nematodes on differentcrops in India. Indian J. Nematol., 37: 219 221.

Kerry, B.R., Kirkwood, I.A. De Leij, F.A.A.M. Barba, J. Leijdens, M.B. and Brookes, P.C. 1993. Growth and survival of Verticillium chlamydosporium Goddard, a parasite of nematodes in soil. Biocontrol Sci. Technol., 3: 355-365.

Latha, T.K.S. and Narashimhan, V. 2001. Management of root rot and cyst nematode complex in black gram. Plant Dis. Res., 16: 73-78.

Muthulakshmi, M. 2011. Biomanagement of potato cyst nematodes, Globodera rostochiensis (wollen weber) and $G$. pallida (stone) using egg parasitic fungi in potato. Ph.D. Thesis, Tamil Nadu 
Agricultural University, Coimbatore, India.

Nagesh, M., Reddy, R.R. Ramachander, N. and Menta, M. 2000. Integrated management of Meloidogyne incongita and Fusarium oxysporum sp. Gladioli in gladiolous using antagonistic fungus and neem cake. In: $3^{\text {rd }}$ International Symposium Nematology Challenges and Opportunities in $21^{\text {st }}$ century conducted Afro-Asian Society of Nematologists (TISAAN) held at Sugarcane Breeding Institute, Coimbatore, pp. 16-19.

Prabhu, S., Kumar, S. Subramanian, S. and Senthil Kumar, P. 2008. Mass production and Commercial formulation of Paecilomyces lilacinus. Indian $J$. Nematol., 38: 131-133.

Rao, M.S. 2005. Management of Meloidogynejavanica on acid lime nursery seedlings by using formulations of Pochonia chlamydosporia and Paecilomyces lilacinus. Nematologia Mediterranea, 33: 145-148.
Sasser, J.N. \& Freckman, D.W. 1987. A world perspectivein Nematology: Role of Society. In: Vistas on Nematology (Ed. I.A. Veech \& D.W. Dickson), pp. 7-14.

Shanthi, A., Rajendran, G. and Sivakumar, M. 2003. Bio-management of lesion nematodes in Banana. In: $6^{\text {th }}$ International workshop on plant growth promoting rhizobacteria, Bangalore, India. pp. 58-63.

Sobita Simon, L. and Avinash Pandey. 2010. Antagonistic efficacy of Paecilomyces lilacinus and Verticillium chlamydosporiun against Meloidogyne incognita infecting okra. Indian $J$. Nematol., 40: 113.

Stirling, G.R. 1991. Biological control of plant parasitic nematodes- Progress, problems and prospects. CAB International, Wallingford, UK. 282p.

http://www.dacnet.nic.in

http://www. tn.gov.in

\section{How to cite this article:}

Muthulakshmi, M., B. Anita and Subramanian, S. 2017. Egg Parasitic Fungus, Engyodontium aranearum with Other Biocontrol Agents, Organic Amendment and Carbofuran for the Management of Meloidogyne incognita on Tomato. Int.J.Curr.Microbiol.App.Sci. 6(5): 29-37. doi: http://dx.doi.org/10.20546/ijcmas.2017.605.004 